Published as: J Am Chem Soc. 2006 June 21; 128(24): 7929-7937.

\title{
Aptamers selected for higher-affinity binding are not more specific for the target ligand
}

\author{
James M. Carothers ${ }^{1}$, Stephanie C. Oestreich², and Jack W. Szostak ${ }^{*}$ \\ Dept. of Molecular Biology, and Center for Computational and Integrative Biology 7215, Simches \\ Research Center, Massachusetts General Hospital, 185 Cambridge Street, Boston, MA 02114
}

\section{Abstract}

Previous study of eleven different in vitro-selected RNA aptamers that bind guanosine

triphosphate (GTP) with $\mathrm{K}_{\mathrm{d}} \mathrm{s}$ ranging from $8 \mu \mathrm{M}$ to $9 \mathrm{nM}$ showed that more information is required to specify the structures of the higher-affinity aptamers. We are interested in understanding how the more complex aptamers achieve higher affinities for the ligand. In vitro selection produces structural solutions to a functional problem that are are as simple as possible in terms of the information content needed to define them. It has long been assumed that the simplest way to improve the affinity of an aptamer is to increase the shape and functional group complementarity of the RNA binding pocket for the ligand. This argument underlies the hypothesis that selection for higher-affinity aptamers automatically leads to structures that bind more specifically to the target molecule. Here, we examined the binding specificities of the eleven GTP aptamers by carrying out competition binding studies with sixteen different chemical analogs of GTP. The aptamers have distinct patterns of specificity, implying that each RNA is a structurally-unique solution to the problem of GTP binding. However, these experiments failed to provide evidence that higher-affinity aptamers bind more specifically to GTP. We suggest that the simplest way to improve aptamer $\mathrm{K}_{\mathrm{d}} \mathrm{s}$ may be to increase the stability of the RNA tertiary structure with additional intramolecular RNA-RNA interactions; increasingly-specific ligand binding may emerge only in response to direct selection for specificity.

\section{Introduction}

RNA aptamers are functional oligonucleotides that fold into distinct three dimensional shapes and bind target ligands ${ }^{1-3}$. In the laboratory, RNA aptamers can be generated by searching large combinatorial pools of random sequences for rare functional molecules using in vitro selection ${ }^{4}$. In vitro selection can yield a diverse array of distinct binding structures which vary significantly in their affinities for the ligand ${ }^{2,5,6}$. With respect to their frequency in sequence space (the ensemble of all possible sequences a particular length) the products of in vitro selection from random sequence pools tend to be the simplest, most abundant, structural solutions to a given problem ${ }^{7}$. For this reason, in vitro-selected aptamers are likely to utilize the least complex structural mechanisms possible to attain the

\footnotetext{
*Corresponding author. Telephone: (617)726-5980 Fax: (617)726-6893. szostak@molbio.mgh.harvard.edu.

${ }^{1}$ Current address: California Institute for Quantitative Biomedical Research and Center for Synthetic Biology, University of California, Berkeley 717 Potter Street MC \#3224, Berkeley, CA 94720-3224

${ }^{2}$ Current address: Novartis Institutes for BioMedical Research, Inc., 400 Technology Square, Cambridge, MA 02139
} 
binding affinities that they exhibit. Therefore, by defining the mechanistic and structural factors that underlie differences in affinity for a set of RNA aptamer structures from the same selection, it should be possible to uncover the simplest ways to improve binding.

It is perhaps easiest to imagine that tighter binding to a target molecule, measured by a lower apparent dissociation constant $\left(\mathrm{K}_{\mathrm{d}}\right)$, emerges through improved shape and functional group complementarity between the ligand and the aptamer binding pocket ${ }^{3,8,9}$. If the binding pocket is rigid, a high degree of interaction and geometric complementarity with the ligand could also be expected to lead to high-specificity binding ${ }^{3,9}$, where specificity is defined as preferential binding to a target ligand compared to other molecules ${ }^{9,10}$. In fact, it has long been assumed that selection for higher-affinity binding automatically leads to aptamers that bind more specifically to a target molecule ${ }^{9}$. However, this view discounts the potential relevance of contributions to the free energy of binding from intramolecular contacts within the RNA itself, as opposed to intermolecular contacts between the RNA and the ligand ${ }^{10}$. To date, this issue has not been explicitly addressed through the analysis of specificity as a function of affinity for a set of aptamers selected against the same target.

The set of 11 different GTP-binding aptamers that we previously isolated ${ }^{5,6}$ provides an ideal testing ground for the affinity-specificity hypothesis (Figure 1, Table 1). The optimized versions of these aptamers vary greatly in sequence and affinity; the $\mathrm{K}_{\mathrm{d}}$ for GTP of the weakest binder is $8 \mu \mathrm{M}$ while the strongest binder in the set has a $K_{d}$ for GTP of $9 \mathrm{nM}$. We used in vitro selection to determine the number of conserved nucleotides and base pairs needed for binding in each aptamer by identifying functional sequence variants of the original isolates ${ }^{6}$. From these data, the information content ${ }^{11-13}$ necessary to specify the aptamer structures in the conditions of the selection was calculated. We found that tighterbinding aptamers are larger, have more intricate secondary structures and are more informationally-complex than weaker-binding aptamers. On average, approximately 10 bits of additional information content, equivalent to specifying 5 more conserved positions or base pairs within an RNA, are required to define structures capable of 10-fold better binding. While this analysis proved that more nucleotide or base-pair positions must be specified in tighter-binding aptamers, there has not yet been a mechanistic or structural explanation for how the more informationally-complex aptamers accomplish higher-affinity binding 6,14 .

Here we present a detailed biochemical characterization of the binding specificities of the set of 11 GTP aptamers. We determined the aptamer affinities for a set of 16 different nucleotide and nucleoside analogs of GTP in competition binding assays and compared these to the aptamer affinities for GTP. First, we find that each aptamer exhibits a unique pattern of ligand-binding specificity compared to the others in the set. Taken together with the fact that there are no sequence motifs common to more than one of the GTP aptamers, this study provides further evidence that each aptamer is a distinct structural solution to the problem of binding GTP with moderate to high-affinity ${ }^{6}$. Furthermore, contrary to expectations ${ }^{9}$, these data do not demonstrate that binding specificity is closely related to binding affinity; aptamers with higher affinities for GTP do not bind more specifically to GTP than aptamers with lower affinities for GTP. We infer that the more complex, higheraffinity aptamers do not make more intermolecular aptamer-ligand functional group 
interactions or have higher degrees of steric complementarity with the ligand. Instead, we propose that much of the observed ${ }^{6}$ informational cost of improving binding reflects the need to increase the number and quality of the contacts within the RNA itself, rather than between the RNA and the ligand.

\section{Experimental Section}

\section{Binding Assays}

The apparent $\mathrm{K}_{\mathrm{d}} \mathrm{s}$ of the aptamers for the GTP analogs were measured in competition binding experiments using a spin filtration method ${ }^{15}$ as previously described ${ }^{5}$ in a buffer of $200 \mathrm{mM} \mathrm{KCl}, 10 \mathrm{mM}$ potassium phosphate, $5 \mathrm{mM} \mathrm{MgCl}_{2}, 0.1 \mathrm{mM}$ EDTA, pH 6.2. RNA was prepared at a concentration of 4 times the $\mathrm{K}_{\mathrm{d}}$ for GTP (final concentration $=2 \times \mathrm{K}_{\mathrm{d}}$ ) in $100 \mu \mathrm{H}_{2} \mathrm{O}$, heated to $80^{\circ} \mathrm{C}$ for $5 \mathrm{~min}$ and then equilibrated overnight at room temperature. Next, $100 \mu \mathrm{l}$ of $2 \times$ binding buffer containing $0.22 \mathrm{nM} \mathrm{a-}{ }^{32} \mathrm{P}-\mathrm{GTP}$ and varying concentrations of unlabeled analog were added. After equilibrating the samples over night at room temperature, they were transferred to the top chamber of a Microcon YM-30 spin filter. The tubes were centrifuged at $13,000 \mathrm{~g}$ for $5 \mathrm{sec}$ to saturate the membrane. The filters were placed into new collection tubes and again spun at 13,000 g for $20 \mathrm{sec}$, allowing $25 \mu \mathrm{l}$ of solution to flow through the membrane. This process is similar to equilibrium dialysis in that the solution that remains above the molecular-weight cutoff membrane contains free GTP $\left([\mathrm{L}]_{\mathrm{f}}\right)$, free analog $\left([\mathrm{I}]_{\mathrm{f}}\right)$, free RNA $\left([\mathrm{R}]_{\mathrm{f}}\right)$, RNA-bound GTP $([\mathrm{RL}])$, and RNA-bound $\operatorname{analog}([R I])$; the filtrate contains only free GTP $\left([L]_{\mathrm{f}}\right)$ and free analog $\left([\mathrm{I}]_{\mathrm{f}}\right)$. A $20 \mu$ sample was removed from each side of the filter, and the radioactivity from the a ${ }^{32} \mathrm{P}-\mathrm{GTP}$ was measured by scintillation counting for $1 \mathrm{~min}$. The radioactive counts were baseline-corrected to account for background retention in the upper chamber of the spin filter ${ }^{16}$. The fraction of labeled GTP bound to the aptamer, $[\mathrm{RL}] /[\mathrm{L}]_{\mathrm{t}}$ (where the total concentration of GTP, $[\mathrm{L}]_{\mathrm{t}}=$ $\left.[R L]+[L]_{f}\right)$, was normalized such that when the concentration of analog competitor was zero, $[R L] /[L]_{t}$ equaled $100 \%$. The $\mathrm{K}_{\mathrm{d}} \mathrm{s}$ were derived from a nonlinear least squares fit of the normalized fraction of labeled GTP bound to the aptamer versus the concentration of analog [I] using R: A Language and Environment for Statistical Computing version 2.0.0 for Linux (R Foundation for Statistical Computing, Vienna, Austria) (see Supporting methods).

\section{Calculating Free Energy of Secondary Structure Formation}

The free energy of aptamer secondary structure formation for the 11 optimized sequences was calculated using the program RNAeval from the ViennaRNA Package version 1.6 for Intel Linux ${ }^{17}$. The optimized sequences were folded at $37^{\circ} \mathrm{C}$ using the default settings with the secondary structure models shown in Figure 1.

To calculate the free energy of secondary structure formation predicted from sequence length, a set of 1000 random sequences was computer generated for each aptamer. The free energy of secondary structure formation for each sequence in the set was then calculated

\footnotetext{
Supporting Information Available Methods for $\mathrm{K}_{\mathrm{d}}$ determination (Supporting methods). Chemical analogs of GTP employed in this study (Supporting Figure 1). Aptamer-analog competition binding curves (Supporting Figure 2). Aptamer patterns of binding specificity (Supporting Figure 3). $\mathrm{K}_{\mathrm{d}}$ (analog) / $\mathrm{K}_{\mathrm{d}}$ (GTP) values for analogs with bearing bulky substituents (Supporting Table 1). $\mathrm{K}_{\mathrm{d} \text { (analog) }} / \mathrm{K}_{\mathrm{d}}$ (GTP) values for analogs with modified or deleted functional groups (Supporting Table 2).
} 
without specifying the secondary structure using the program RNAfold from the ViennaRNA package version 1.6. Sequences were folded at $37^{\circ} \mathrm{C}$ using the default settings. In the same manner, 1000 sets of 11 aptamer-length sequences were generated and folded using RNAfold to estimate the likelihood that all of the aptamers in the set would, by chance, have free energies of secondary structure formation more stable than the median values for same-length random sequences.

\section{Correlation coefficients and regressions}

The Spearman rank correlation coefficient $\left(\mathrm{r}_{\mathrm{s}}\right)$ was applied as a nonparametric test of association, since it does not require specific assumptions about data distribution and is insensitive to deviations from the normal distribution. ${ }^{18} 95 \%$ confidence intervals were used for all rankings and significance was determined under the null hypothesis of no correlation with a one-tailed test, $a=0.05 . P$ values were calculated from the test statistic as described ${ }^{19}$. To quantify the number of aptamer-analog pairs with $\log _{10} \mathrm{~K}_{\mathrm{d} \text { (analog) }} / \mathrm{K}_{\mathrm{d} \text { (GTP) }}$ values at a given threshold, significance was determined with the $\mathrm{K}_{\mathrm{d}} \mathrm{s}$ and standard deviations obtained from nonlinear least square fits of the analog binding data under the null hypothesis of no difference with a two-tailed test, $\alpha=0.05$ (Note: none of the major conclusions would be different if smaller a values had been used). Kendall's robust line-fit method is a nonparametric approach to curve fitting that does not require independent and normally-distributed data ${ }^{19}$. The slope is given by the median slope of all pairwise combinations of points. The intercept is the median intercept of all points computed with the median slope.

\section{Results}

\section{Measuring aptamer specificity for GTP}

In order to determine the extent to which the aptamers (Figure 1) bind specifically to GTP, we measured their affinities for two different sets of chemical analogs of GTP (Figure 2). Aptamer affinities for each analog were obtained from competition binding assays with radiolabeled ${ }^{32} \mathrm{P}-\mathrm{GTP}$ and unlabeled analog. Details of the assays are described in the Experimental Section and the supporting information.

The 10 analogs in the first set (Figure 2A) have bulky substituents, and were chosen to provide a way of screening for close approaches between the aptamer RNA and the GTP ligand. The set consists of six different methylated analogs, 8-amino-guanosine, and three analogs in which a larger sulfur atom replaces an oxygen atom: 6-thio GTP, GTP-a-S and GTP- $\gamma$-S. Such bulky analogs could have altered binding affinity compared to GTP for one, or more, of the following reasons. There may be drastic reductions in binding affinity (increases in $\mathrm{K}_{\mathrm{d}}$ ) if the aptamer cannot sterically accommodate the bulky substituent without radically restructuring the binding pocket. Relative to GTP, 3-methyl-guanosine and 7methyl-guanosine have potential hydrogen bond acceptors that are blocked; the hydrogenbonding ability of the sulfur-substituted analogs is also reduced ${ }^{20}$. Other analogs, $2^{\prime} \mathrm{O}-$ methyl-GTP, 3'O-methyl-GTP, 1-methyl-guanosine, and N2-methyl-2'-deoxy-GTP, have potential hydrogen bond donors that are eliminated. If the potential hydrogen bond donor or acceptor is utilized as such in the aptamer-ligand complex, its absence should have a 
negative effect on affinity. Finally, with the exception of the sulfur-containing analogs, the addition of the bulky substituents changes the hydrophobicity of the ligand, where the effect on the binding affinity could be either positive or negative ${ }^{21}$.

The 6 molecules in the second set of analogs (Figure 2B) are characterized by the removal or alteration of individual functional groups. These modifications include the loss of phosphate groups, sugar hydroxyls, or the exocyclic amine, or change of N7 in the nucleobase to $\mathrm{CH}$. Here, a difference in binding affinity for one of these analogs compared to GTP captures the net energetic effect of changes in solvation, hydrogen bonding, and van der Waals and electrostatic interactions.

The apparent dissociation constant $\left(\mathrm{K}_{\mathrm{d}}\right)$ for an aptamer-ligand complex describes the ratio of the concentration of the complex to the concentrations of free aptamer and free ligand at equilibrium. Explicitly, $\mathrm{K}_{\mathrm{d}}=[\mathrm{RNA}][$ Ligand]/[RNA:Ligand], where the equilibrium constant is directly related to the change in standard free energy, $\Delta \mathrm{G}=-\mathrm{RT} \ln 1 / \mathrm{K}_{\mathrm{d}}{ }^{22}$. Specificity can be measured as the difference in the free energy of binding to one ligand (L) versus another $\left(\mathrm{L}^{\prime}\right)^{9,10}$ :

$$
\Delta \Delta \mathrm{G}=\Delta \mathrm{G}_{\mathrm{L}}-\Delta \mathrm{G}_{\mathrm{L}}=-\mathrm{RT} \ln \frac{\mathrm{K}_{\mathrm{d}\left(\mathrm{L}^{\prime}\right)}}{\mathrm{K}_{\mathrm{d}(\mathrm{L})}}
$$

Taking

$$
\log _{10} \frac{\mathrm{K}_{\mathrm{d}(\text { analog })}}{\mathrm{K}_{\mathrm{d}(\mathrm{GTP})}}
$$

quantifies the magnitude by which an aptamer binds specifically to GTP compared to an analog. For example, the 10-10 aptamer $\mathrm{K}_{\mathrm{d}}$ for GTP is $30 \pm 6 \mathrm{nM}$ (Table 1), while its $\mathrm{K}_{\mathrm{d}}$ for 2 -deoxy-GTP $(6500 \pm 200 \mathrm{nM})$ is more than two orders of magnitude higher $\left(\log _{10}\right.$ $\mathrm{K}_{\mathrm{d}(2 ' \text {-deoxy-GTP) }} / \mathrm{K}_{\mathrm{d}(\mathrm{GTP})}=2.3$ ) (Table 2, Supporting Figure 1C). In other words, the 10-10 aptamer favors binding to GTP more than 200 fold compared to 2'-deoxy-GTP.

Equivalently, the 10-10 specificity for GTP compared to 2'-deoxy-GTP could be expressed as $\Delta \Delta \mathrm{G}=3.2 \mathrm{kcal} \mathrm{mol}^{-1}$ at $25^{\circ} \mathrm{C}$. In contrast, the 9-4 aptamer $\mathrm{K}_{\mathrm{d}}$ for 2'-deoxy-GTP $(10 \pm 3$ $\mathrm{nM}$ ) (Supporting Figure 1A) is the same as the 9-4 aptamer $\mathrm{K}_{\mathrm{d}}$ for GTP $(9 \pm 1 \mathrm{nM})$ (Table 1). Hence, for the 9-4 aptamer, $\log _{10} \mathrm{~K}_{\mathrm{d} \text { (2'-deoxy-GTP) }} / \mathrm{K}_{\mathrm{d} \text { (GTP) }}$ and the corresponding $\Delta \Delta \mathrm{G}$ are close to zero, indicating that the 9-4 aptamer does not bind specifically to GTP compared to 2'-deoxy-GTP.

The $\log _{10} \mathrm{~K}_{\mathrm{d} \text { (analog) }} / \mathrm{K}_{\mathrm{d} \text { (GTP) }}$ values for all of the aptamers are plotted in Figure 3 (also see Tables 2 and 3 and supporting Table 1). For $34 \%$ of the aptamer-analog pairs, aptamer binding was not very specific for GTP relative to the analog; $\log _{10} \mathrm{~K}_{\mathrm{d} \text { (Analog) }} / \mathrm{K}_{\mathrm{d} \text { (GTP) was }}$ $<1$ and $\Delta \Delta \mathrm{G}<1.4 \mathrm{kcal} \mathrm{mol}^{-1}$. For $38 \%$ of the aptamer-analog pairs, aptamer binding was

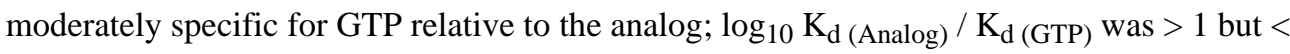
$2\left(1.4 \mathrm{kcal} \mathrm{mol}^{-1}<\Delta \Delta \mathrm{G}<2.7 \mathrm{kcal} \mathrm{mol}^{-1}\right)$. In the remaining $28 \%$ of the aptamer-analog pairs, binding was very specific for GTP, with $\log _{10} \mathrm{~K}_{\mathrm{d} \text { (Analog) }} / \mathrm{K}_{\mathrm{d} \text { (GTP) }}>2(\Delta \Delta \mathrm{G}>2.7$ kcal mol$\left.{ }^{-1}\right)$. For many of these cases the ${ }^{32} \mathrm{P}-\mathrm{GTP}$ was not detectably displaced in the 
competition binding assays, i.e. no binding was detected, corresponding to $\log _{10} \mathrm{~K}_{\mathrm{d}}$ (Analog) / $\mathrm{K}_{\mathrm{d} \text { (GTP) }} \gg>3$ except in the case of the low affinity Class III aptamer, for which no binding corresponds to $\log _{10} \mathrm{~K}_{\mathrm{d} \text { (Analog) }} / \mathrm{K}_{\mathrm{d}(\mathrm{GTP})} \gg 2$.

Overall, the aptamers have very little specificity for the ligand triphosphate region, and only slightly more discrimination for modifications to the ribose sugar. All of the aptamers are clearly most specific for the GTP nucleobase (Figure 2).

Only the Class III aptamer showed greatly reduced binding $\left(\log _{10} \mathrm{~K}_{\mathrm{d}(\text { Analog) }} / \mathrm{K}_{\mathrm{d}(\mathrm{GTP})}>2\right)$ to analogs where the triphosphate had been modified or deleted. The Class III aptamer does not bind to guanosine even though the Class III aptamer $\mathrm{K}_{\mathrm{d}}$ s for GDP, GMP, GTP- $\gamma-\mathrm{S}$ and GTP-a-S are nearly the same as the $\mathrm{K}_{\mathrm{d}}$ for GTP (Tables 1 and 2). Note that due to limited analog solubility, we could not accurately measure the Class III aptamer affinity for any analog where the $\mathrm{K}_{\mathrm{d}}$ was $>2$ orders of magnitude larger than the Class III aptamer $\mathrm{K}_{\mathrm{d}}(8 \mu$ M) for GTP. Nonetheless, we can conclude that the Class III aptamer $K_{d}$ for guanosine is at least two orders of magnitude worse than the Class III $\mathrm{K}_{\mathrm{d}}$ for GTP.

Across the entire set, the aptamers display slightly more specificity for the ligand ribose sugar than the triphosphate region. Only two of the aptamers had >100-fold increases in $\mathrm{K}_{\mathrm{d}}$ when the 2' $\mathrm{OH}$ was removed (Table 2) and none were affected that strongly by loss of the $3^{\prime}$ OH. Aptamers 10-10, 10-6 and Class IV bound poorly to 2'-O-methyl-GTP and neither the Class IV nor the Class III aptamers exhibited binding to 3'-O-methyl-GTP.

Modifications to the nucleobase moiety (Figure 2) caused by far the greatest effects on binding. In fact, just $25 \%$ (19 of 77) of the aptamer-analog combinations involving analogs with nucleobase changes or deletions exhibited binding within 100-fold that of GTP ( $\log _{10}$ $\mathrm{K}_{\mathrm{d}(\text { Analog) }} / \mathrm{K}_{\mathrm{d}(\mathrm{GTP})}<2$ ). When free in solution, guanosine nucleotides and nucleosides typically adopt syn conformations about their glycosidic bonds, in contrast to most other nucleotides and nucleosides that adopt anti conformations ${ }^{23}$. Inosine triphosphate (ITP) differs from GTP only in the loss of the 2-amino group, one effect of which is that ITP exists in the anti conformation ${ }^{23}$. The importance of the glycosidic bond conformation and the sensitivity of the selected aptamers to modifications on the ligand nucleobase are illustrated by the fact that none of the aptamers bind detectably to ITP, or to ATP, CTP, TTP or UTP (data not shown).

\section{Distinct patterns of binding specificity}

We used the results from the competition binding assays to diagram the pattern of binding specificity for each aptamer (Tables 2 and 3; Figure 4). Ligand positions where modification resulted in substantially-reduced binding are denoted by red shading in Figure 4.

Interestingly, each aptamer displays a pattern of binding specificity that is different from the other aptamers in the set. Here, specific binding to GTP compared to a given analog was demarcated by a $\log _{10} \mathrm{~K}_{\mathrm{d} \text { (Analog) }} / \mathrm{K}_{\mathrm{d}(\mathrm{GTP})}$ value $>2$.

Changing the cut-off used to signify specific binding to, for example, $\log _{10} \mathrm{~K}_{\mathrm{d} \text { (Analog) } /}$ $\mathrm{K}_{\mathrm{d} \text { (GTP) }}>1$, alters the patterns illustrated in figure 4. However, even with different cut-offs to signify specific binding, the aptamers appear sensitive to distinct subsets of ligand 
modifications (also see Supporting Figure 3). We interpret this to mean that each aptamer has a distinct ligand-binding surface.

\section{Affinity and Specificity}

We sought evidence that aptamers with high affinity for GTP are also more specific for GTP binding than aptamers with low affinity for GTP. We expected that aptamers with high affinity (lower $\mathrm{K}_{\mathrm{d}}$ ) for GTP would bind well to a smaller number of analogs than aptamers with low affinity (higher $\mathrm{K}_{\mathrm{d}}$ ) for GTP. For each aptamer we plotted the number of analogs to which the RNA exhibited high-affinity binding relative to GTP against the aptamer $\mathrm{K}_{\mathrm{d}}$ for GTP (Figure 5). Initially, we examined analogs with $\log _{10} \mathrm{~K}_{\mathrm{d} \text { (Analog) }} / \mathrm{K}_{\mathrm{d} \text { (GTP) }}$ values $<2$ (colored red in Figure 5). All of the aptamers displayed binding in this range to 8 to 12 of the analogs. Surprisingly, we do not see any evidence that the number of analogs to which each aptamer binds in this range is correlated with binding affinity for GTP $\left(r_{s}=0.07\right.$, $P=0.42$ ).

In order to ensure that choosing $\log _{10} \mathrm{~K}_{\mathrm{d} \text { (Analog) }} / \mathrm{K}_{\mathrm{d} \text { (GTP) }}<2$ as a cut-off did not obscure more subtle differences in specificity, we also computed the correlation statistic using $\log _{10}$ $\mathrm{K}_{\mathrm{d} \text { (Analog) }} / \mathrm{K}_{\mathrm{d} \text { (GTP) }}<1$ and $<3$ as the cut-offs (Figure 5). There was no significant change in the correlation statistic whether the $\log _{10} \mathrm{~K}_{\mathrm{d} \text { (Analog) }} / \mathrm{K}_{\mathrm{d} \text { (GTP) }}$ cut-off for specific binding was $<1,<2$ or $<3$. Thus, we do not find that aptamers that bind with more affinity for GTP are more specific for GTP binding compared to chemically-related analogs.

\section{Free energy of secondary structure formation}

Within the set of selected aptamers, the higher-affinity molecules tend to have longer sequences, are more informationally-complex and have more intricate secondary structures ${ }^{6}$ (Figure 1; Table 1). It is known that selection for more stable RNA tertiary structures can produce RNAs with more stable secondary structures ${ }^{24}$. We wondered whether selection for tighter-binding yields aptamers with unusually-stable secondary structures.

We calculated the free energy of secondary structure formation using the program RNAeval ${ }^{17}$ (Table 1). There is a significant correlation $(P<0.02)$ between the apparent $\mathrm{K}_{\mathrm{d}}$ for GTP and the free energy of secondary structure formation $\left(\Delta \mathrm{G}_{2}{ }^{\circ}\right.$ (folding) (Figure 6A). We compared the calculated $\Delta \mathrm{G}_{2^{\circ}}$ (folding) value for each aptamer with 1000 computergenerated random sequences of the same length (Figure 6B). All eleven aptamers have $\Delta \mathrm{G}_{2}$ (folding) values more stable than the median same-length random sequence values. The likelihood that eleven aptamer-length sequences would have calculated $\Delta \mathrm{G}_{2}^{\circ}$ (folding) values larger than the median by chance is very small $(P<0.001)$, suggesting that selection for secondary structural stability may have taken place. However, only the 9-4, Class V, 10-59 and Class III aptamers have calculated free energies of secondary structure formation that are significantly more stable than the same-length random sequences (i.e. $\Delta \mathrm{G}_{2}{ }^{\circ}$ (folding) values more stable than $95 \%$ of the same-length random sequence values).

It is possible that the calculated $\Delta \mathrm{G}_{2^{\circ}}$ (folding) values do not accurately capture the magnitude of the actual secondary structure free energies because they do not consider the potential influence of the ligand or tertiary interactions within the RNA. Still, to a first approximation, 
these results indicate that selection for higher-affinity binding leads to RNAs with more stable secondary structures, but not necessarily to aptamers with secondary structures that are substantially more stable than predicted on the basis of size.

\section{Discussion}

We investigated the relationship between RNA aptamer binding affinity and binding specificity for a target ligand. The test set of evolved aptamers consisted of molecules with $\mathrm{K}_{\mathrm{d}} \mathrm{s}$ for GTP that range from $8 \mu \mathrm{M}$ to $9 \mathrm{nM}$. We assessed the affinity of each of the aptamers for a series of sixteen different chemical analogs of GTP, along with ATP, CTP, UTP, TTP and ITP in competition binding assays. Aptamer specificity for GTP was quantified as $\log _{10}$

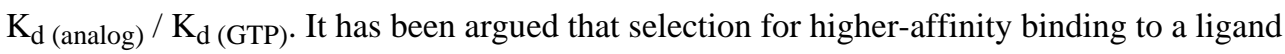
automatically leads to aptamers with more specificity for the ligand ${ }^{9}$. Surprisingly, we did not find any evidence that the higher-affinity aptamers are more specific for GTP than the lower-affinity aptamers (Table 1; Figures 3 and 5).

We examined a wide range of degrees of specificity in order to maximize our chances of observing an affinity-specificity correlation. We initially set the cut-off for significant differences in specificity as $\log _{10} \mathrm{~K}_{\mathrm{d} \text { (analog) }} / \mathrm{K}_{\mathrm{d} \text { (GTP) }}>2$ because previous studies have suggested that differences of that magnitude could be attributed to readily-observable structural features of the aptamer-ligand complex such as a steric clash or loss of a hydrogen bond ${ }^{15,21,25-27}$. Solution NMR studies of the Class I GTP aptamer ${ }^{28}$ demonstrate that analogs for which $\log _{10} \mathrm{~K}_{\mathrm{d} \text { (Analog) }} / \mathrm{K}_{\mathrm{d} \text { (GTP) }}>2$ have modifications that introduce discernible steric clashes and/or the loss of hydrogen-bonding interactions with the aptamer. Likewise, biochemical and structural ${ }^{15,27}$ studies of an RNA aptamer that binds theophylline showed that ligand modifications that result in approximately 100 fold or greater increases in $\mathrm{K}_{\mathrm{d}}$ relative to theophylline correspond to particular intermolecular aptamer-ligand contacts. As noted above, we could not measure the Class III aptamer affinity for any analog whose $\mathrm{K}_{\mathrm{d}}$ was $>100$ fold higher than its $\mathrm{K}_{\mathrm{d}}$ for GTP $(8 \mu \mathrm{M})$ because of limited analog solubility. Therefore, both empirical observations of aptamer structures and practical considerations indicate that the most conservative treatment of the data was to use $\log _{10}$ $\mathrm{K}_{\mathrm{d} \text { (Analog) }} / \mathrm{K}_{\mathrm{d} \text { (GTP) }}>2$ as the cut-off for meaningful differences in binding specificity. However, using $\log _{10} \mathrm{~K}_{\mathrm{d} \text { (Analog) }} / \mathrm{K}_{\mathrm{d} \text { (GTP) }}$ cut-offs of $>1$ or $>3$ did not lead to either quantitatively or qualitatively different outcomes (Fig. 5).

The GTP analogs that we tested for aptamer binding had bulky substituents and functional group substitutions and deletions in the nucleobase, sugar and triphosphate regions. It is possible that differences in binding specificity are limited to sites for which we could not screen the effect of chemical group modifications, such as the sugar 1' and 4' positions or the nucleobase 4 and 5 positions. There were also many cases where the analogs bound so poorly that the binding affinity could only be described qualitatively as 'no binding detected'. Neither of these caveats alters the overall picture: there is no obvious trend toward substantially more binding specificity for GTP as the aptamer affinity for GTP increases.

It is notable that all of the aptamers in our set have unique patterns of ligand-binding specificity (Figure 4; Supporting Figure 3). NMR structural studies of the Class I GTP 
aptamer ${ }^{28}$ illustrate that the RNA-ligand contacts are mediated by platforms of aptamer purines that sandwich the planar portion of the ligand and a base-base interaction between the GTP and an aptamer guanosine. This general mode of recognition was seen previously in RNA aptamers that bind to $\mathrm{ATP}^{25,26,29}, \mathrm{FMN}^{30,31}$, and theophylline ${ }^{3,15,27}$. Further high resolution structural studies will be required to determine whether this motif is present in any of the other GTP aptamers. Although some of the GTP aptamers may employ common modes of ligand binding, the fact that they have unique patterns of binding specificity implies that there are differences in the shapes and compositions of their binding pockets. These results, combined with the observation that the GTP aptamer recognition bulges do not share sequence identity ${ }^{6}$ (Figure 1), reinforce the notion that large sets of structurallydiverse aptamers can be recovered from sparse searches of sequence space ${ }^{5,6}$.

The GTP aptamers were isolated from pools of molecules containing long stretches of random sequence ${ }^{5}$. The aptamers most likely to be recovered are the simplest structures, defined as those lowest in informational complexity, with the binding affinity required to survive the process of selection ${ }^{7,32}$. Therefore, the physical mechanisms used to attain the required $\mathrm{K}_{\mathrm{d}} \mathrm{s}$ should be the simplest to implement structurally. Earlier efforts to understand the mechanisms by which aptamers achieve low $\mathrm{K}_{\mathrm{d}} \mathrm{s}$ focused exclusively on the energetics of the local intermolecular aptamer-ligand interactions ${ }^{3,9}$. Those analyses assumed that maximizing the intermolecular geometric complementarity and the quality and quantity of aptamer-ligand functional group interactions within the binding pocket was the easiest way to improve the aptamer $\mathrm{K}_{\mathrm{d}}$ for a ligand (e.g. aligning hydrogen-bond donors within the RNA with hydrogen-bond acceptors in the ligand). All else being equal, increased aptamer-ligand steric and functional group complementarity would cause higher-affinity aptamers to bind more specifically to the target ligand than lower-affinity aptamers ${ }^{9}$. Conversely, since we found that higher-affinity aptamers do not bind more specifically to the target ligand (Table 1, Figure 5) we conclude that their binding pockets are unlikely to have better shape and functional group complementarity with the ligand than the binding pockets of lower-affinity aptamers.

How might aptamers achieve higher-affinity binding without an increased number of intermolecular interactions and better shape complementarity with the ligand? Obviously, the local energetics of the aptamer-ligand interaction are an important aspect of the binding affinity ${ }^{3,8,9}$. However, the aptamer-ligand $\mathrm{K}_{\mathrm{d}}$ is determined by the $\Delta \mathrm{G}$ between the bound and unbound states of the entire system, not just the local aptamer-ligand interactions ${ }^{10}$. In fact, structural studies have revealed that ligand binding and RNA aptamer folding are often tightly coupled ${ }^{3,26-28,33}$, underscoring the potential relevance of tertiary intramolecular RNA-RNA interactions in improving the aptamer-ligand $\mathrm{K}_{\mathrm{d}}$. We hypothesize that differences in the tertiary stabilities of the RNA folds could account for much of the observed variation in aptamer-GTP $\mathrm{K}_{\mathrm{d}}$ s. If so, the same physical mechanisms that improve the aptamer $\mathrm{K}_{\mathrm{d}}$ for the target ligand would also improve the aptamer $\mathrm{K}_{\mathrm{d}}$ for chemicallyrelated ligands. In turn, this would explain why we do not see evidence that higher-affinity aptamers bind more specifically to GTP.

There is a positive correlation between aptamer-GTP $\mathrm{K}_{\mathrm{d}}$ and the calculated $\Delta \mathrm{G}$ of secondary structure formation (Figure 6A). This is consistent with a role for the stability of the RNA 
secondary structure fold in differentiating the higher-affinity from the lower-affinity aptamers. Yet, only four of the eleven aptamers (Figure 6B) have calculated $\Delta \mathrm{Gs}$ of secondary structure formation that are significantly more stable than predicted from their sequence lengths. One possibility is that while the higher-affinity aptamers could have more stable tertiary folds, there was no direct selection pressure for more stable secondary structures ${ }^{34}$. Because RNA tertiary folding is highly-cooperative and facilitated by non-local interactions ${ }^{10}$ it is difficult to separate the ligand binding energies from RNA folding energies except where only simple base-pairing is involved ${ }^{35}$. Here the analysis was restricted to consideration of the calculated free energies of secondary structure formation. Clearly, high resolution structure models will be necessary to fully understand the role of the RNA-RNA interactions, separate from the RNA-ligand interactions, in determining tertiary structural stability and aptamer-ligand affinities.

We know from sequence comparison data that more nucleotides in the higher-affinity GTP aptamers are subject to functional constraints ${ }^{6}$. The functional constraints are a consequence of the need to make intermolecular contacts with the ligand in the context of a folded RNA secondary and tertiary structure. For this set of aptamers, the informational cost to improve binding affinity 10 -fold is approximately 10 bits, enough to specify 5 conserved positions or 5 base pairs in an RNA structure in the conditions of the original selection ${ }^{5}$. The findings presented here suggest that increasing the RNA shape and functional group complementarity for the ligand is not the simplest way to improve binding affinity. Rather, the informational cost of improving aptamer affinity may be a function of the increased complexity required to form more stable tertiary RNA structures.

One prediction arising from the suggestion that higher-affinity binding may be achieved through the formation of more stable tertiary structures is that selection for stability could inadvertently result in molecules that are more specific for the target ligand. Selection for stability could produce a molecule with more rigidity in the elements surrounding a binding pocket, eliminating the structural flexibility needed to accommodate chemically-related analogs of a given ligand ${ }^{36}$. Consistent with this expectation, a non-biological ATP binding protein subjected to selection for increased stability in the presence of a chemical denaturant showed more specific binding to ATP when tested with a panel of analogs ${ }^{36}$.

We have previously suggested ${ }^{6}$ that RNA aptamers that bind planar, polar ligands, such as GTP, require less sequence information to specify than structures that bind flexible, nonpolar targets with the same affinity in similar environments. We further argued that a particular degree of improvement in binding affinity could have the same informational cost, regardless of the chemical nature of the ligand, if increasing the number of interactions within the RNA, rather than between the RNA and the ligand, is generally the simplest way to improve affinity. Determining whether the observations and conclusions made here about GTP can be extended to other non-planar, or non-polar ligands will be an important test of this hypothesis.

Increasing aptamer affinity by stabilizing the tertiary fold of the RNA, or by improving the quality of the interactions with the ligand, do not seem mutually exclusive. Why then is more specific binding, predicted from the latter process, not observed in our set of aptamers? 
We propose several potentially relevant factors. Across the whole set of aptamers, (Figures 2 and 4) binding is greatly affected by modifications to the ligand nucleobase. It may be difficult for additional RNA-ligand nucleobase interactions to come into play. At the same time, modifications to the ligand ribose or triphosphate frequently have only small negative effects on binding. Since the aptamers were selected from a random sequence pool, we only see the most likely solutions. Improving aptamer $\mathrm{K}_{\mathrm{d}}$ by increasing the tertiary stability of the RNA must require structures that are less informationally-complex than those needed to improve aptamer $K_{d}$ by interacting more strongly with the sugar or triphosphate moieties of the ligand ${ }^{37}$. Although higher-affinity aptamers that bind more specifically to the target ligand may exist in sequence space, their greater informational complexities mean that they can only be isolated by selecting for both affinity and specificity.

\section{Acknowledgments}

We thank G. Cho for help with the competition binding curve analysis and A. Keefe and R. Larralde for many useful suggestions. We thank B. Seelig for comments on the manuscript. This work was supported by a grant from the US National Institutes of Health (GM53936 to J.W.S.). J.M.C. was supported in part by a US National Science Foundation Graduate Fellowship. S.C.O. was supported in part by a PhD scholarship of the Foundation of the German Chemical Industry. J.W.S. is an Investigator of the Howard Hughes Medical Institute.

\section{References}

1. Ellington AE, Szostak JW. Nature. 1990; 346:818-822. [PubMed: 1697402]

2. Wilson DS, Szostak JW. Annu. Rev. Biochem. 1990; 68:611-647. [PubMed: 10872462]

3. Hermann T, Patel DJ. Science. 2000; 287:820-825. [PubMed: 10657289]

4. Carothers, JM.; Szostak, JW. The aptamer handbook: functional oligonucleotides and their applications. Klussmann, S., editor. Berlin: Wiley-VCH; 2006. p. 3-28.

5. Davis JD, Szostak JW. Proc. Natl. Acad. Sci. U.S.A. 2002; 99:11616-11621. [PubMed: 12185247]

6. Carothers JM, Oestreich SC, Davis JH, Szostak JW. J. Am. Chem. Soc. 2004; 126:5130-5135. [PubMed: 15099096]

7. Lorsch JR, Szostak JW. Acc. Chem. Res. 1996; 29:103-110. [PubMed: 11539421]

8. Lancet D, Sadovsky E, Seidemann E. Proc. Natl. Acad. Sci. U.S.A. 1993; 90:3715-3719. [PubMed: 8475121]

9. Eaton BE, Gold L, Zichi DA. Chem. \& Biol. 1995; 2:633-638. [PubMed: 9383468]

10. Szwajkajzer D, Carey J. Bioploymers. 1997; 44:181-198.

11. Schneider TD, Stormo GD, Gold L, Ehrenfeucht A. J. Mol. Biol. 1986; 188:415-431. [PubMed: 3525846]

12. Adami C, Cerf NJ. Physica D (Amsterdam). 2000; 37:62-69. 2000.

13. Adami C. Physics of Life Reviews. 2004; 1:3-22.

14. Lau MWL, Cadieux KEC, Unrau PJ. J. Am. Chem. Soc. 2004; 126:15686-15693. [PubMed: 15571390]

15. Jenison RD, Gill SC, Pardi A, Polisky B. Science. 1994; 263:1425-1429. [PubMed: 7510417]

16. Motulsky, H.; Christopoulos, A. Fitting Models to Biological Data using Linear and Nonlinear Regression. San Diego, CA: Graphpad; 2003.

17. Hofacker IL, Fontana W, Stadler PF, Bonhoeffer LS, Tacker M, Schuster P. Monatshefte f. Chemie. 1994; 125:167-188.

18. Sokol, RR.; Rohlf, J. Biometry: The Principals and Practice of Statistics in Biological Research. 3rd ed.. New York, NY: W. H. Freeman \& Co.; 1995.

19. Rosner, BA. Fundamentals of Biostatistics. 4th ed.. Belmont, CA: Duxbury Press; 1995.

20. Christian EL, Yarus M. J. Mol. Biol. 1992; 228:743-758. [PubMed: 1469712]

21. Koizumi M, Breaker RR. Biochemistry. 2000; 39:8983-8992. [PubMed: 10913311] 
22. Eisenberg, D.; Crothers, DM. Physical Chemistry with Applications to the Life Sciences. Menlo Park, CA: Benjamin; 1979.

23. Saenger, W. Principles of nucleic acid structure. New York, NY: Springer-Verlag New York; 1984.

24. Juneau K, Podell E, Harrington DJ, Cech TR. Structure (Camb). 2001; 9:221-231. [PubMed: 11286889]

25. Sassanfar M, Szostak JW. Nature. 1993; 364:550-553. [PubMed: 7687750]

26. Dieckmann T, Suzuki E, Nakamura GK, Feigon J. RNA. 1996; 2:628-640. [PubMed: 8756406]

27. Zimmermann GR, Jenison RD, Wick CL, Simorre JP, Pardi A. Nat. Struct. Biol. 1997; 4:644-649. [PubMed: 9253414]

28. Carothers JM, Davis JH, Chou JJ, Szostak JW. RNA. 2006; 12:567-579. [PubMed: 16510427]

29. Jiang F, Kumar RA, Jones RA, Patel DJ. Nature. 1996; 382:183-186. [PubMed: 8700212]

30. Burgstaller P, Famulok M. Angew. Chem. Int. Ed. Engl. 1994; 33:1084-1087.

31. Fan P, Suri A, Fiala R, Live D, Patel D. J. Mol. Biol. 1996; 258:480-500. [PubMed: 8642604]

32. Salehi-Ashtiani K, Szostak JW. Nature. 2001; 414:82-84. [PubMed: 11689947]

33. Sussman D, Nix JC, Wilson C. Nat. Struct. Biol. 2000; 7:53-57. [PubMed: 10625428]

34. Guo F, Ceck TR. Nat. Struct. Biol. 2002; 9:855-861. [PubMed: 12368901]

35. Hertel KJ, Peracchi A, Uhlenbeck OC, Herschlag D. Proc. Natl. Acad. Sci. U.S.A. 1997; 94:84978502. [PubMed: 9238005]

36. Chaput JC, Szostak JW. Chem. \& Biol. 2004; 11:865-874. [PubMed: 15217619]

37. Sazani PL, Larralde R, Szostak JW. J. Am. Chem. Soc. 2004; 126:8370-8371. [PubMed: 15237981] 


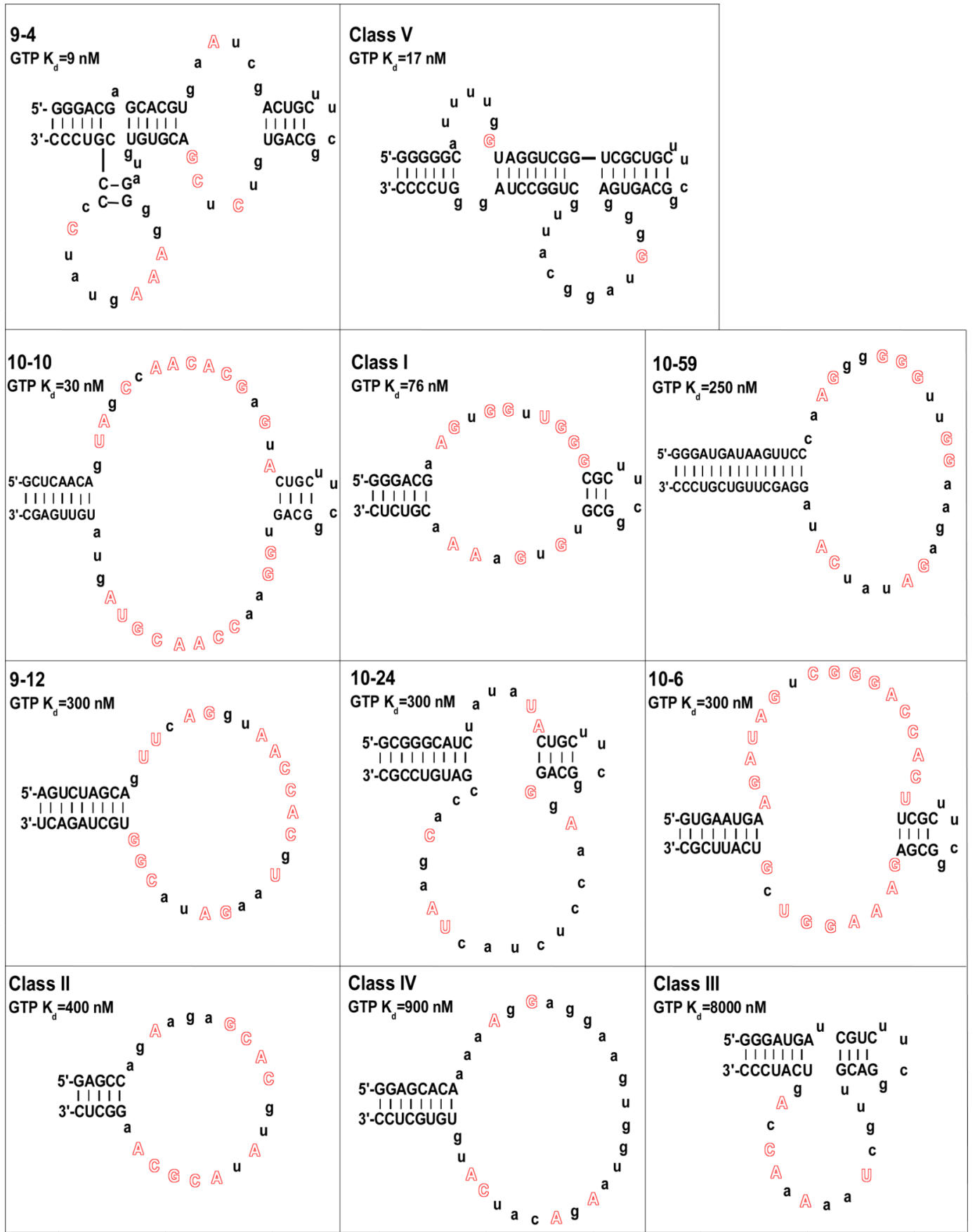

Figure 1.

GTP aptamer secondary structures. The secondary structure of each GTP aptamer used in this analysis is shown with invariant positions in the recognition loops outlined in red. 


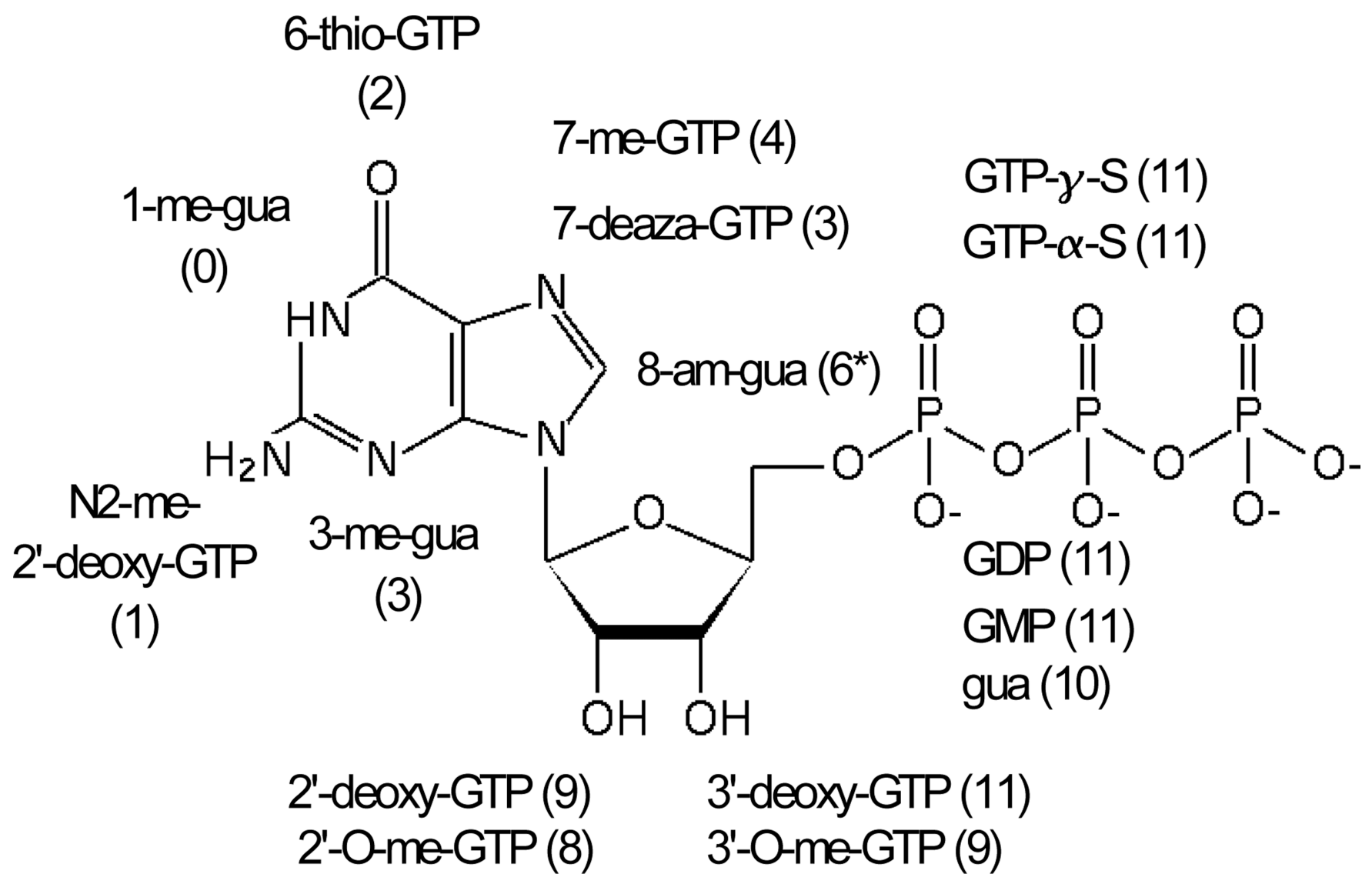

Figure 2.

GTP functional groups and their importance for aptamer binding. The role of each functional group in binding was tested by comparison of analog and GTP binding affinities. The number of aptamers (out of 11 total) that suffered no more than a 100-fold loss in affinity to the analog $\left(\log _{10} \mathrm{~K}_{\mathrm{d} \text { (Analog) }} / \mathrm{K}_{\mathrm{d} \text { (GTP) }}<2\right)$ is shown in parentheses next to each functional group. Functional groups on the nucleobase play a more significant role in aptamer binding than functional groups on the sugar and triphosphate. 

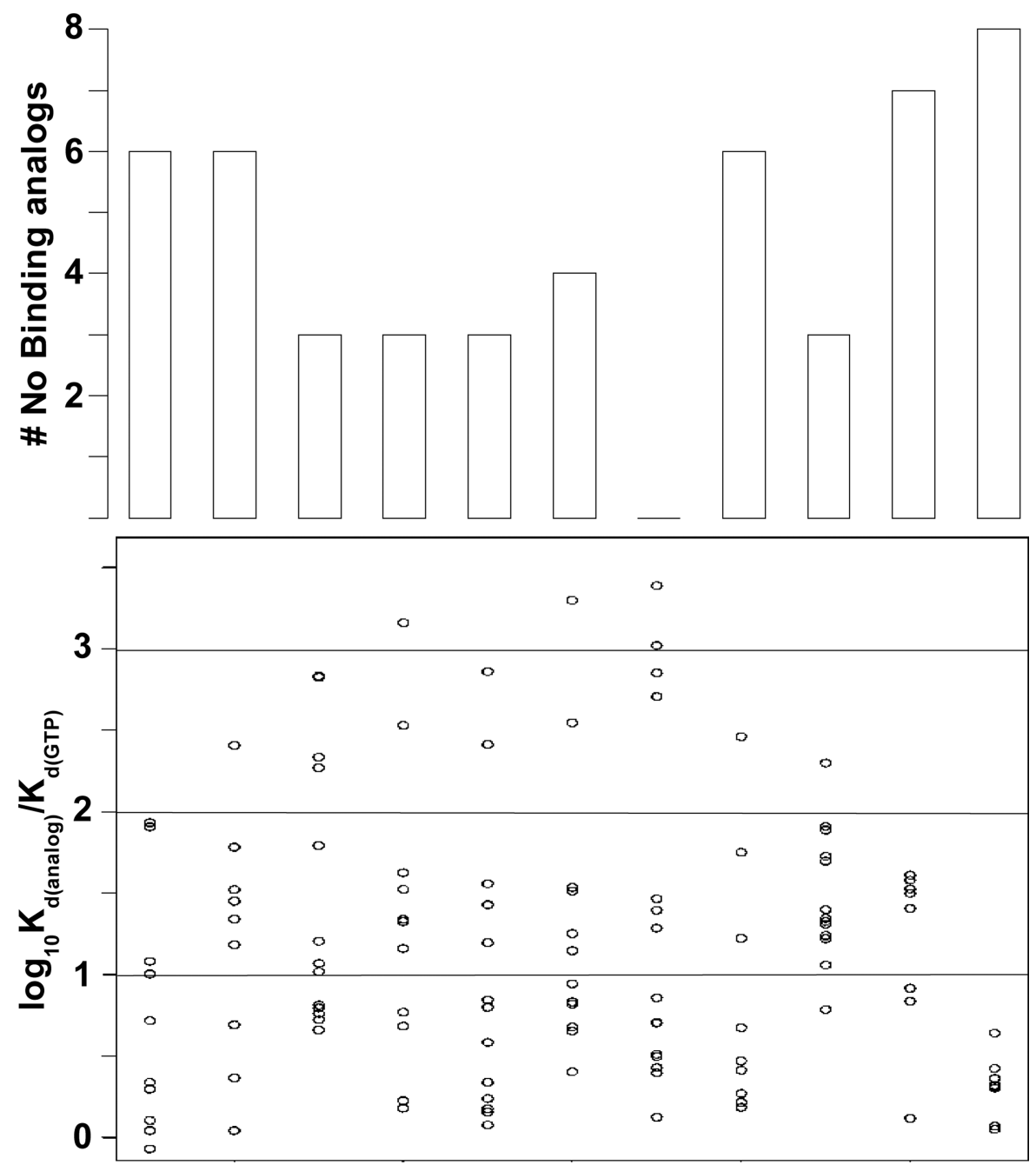

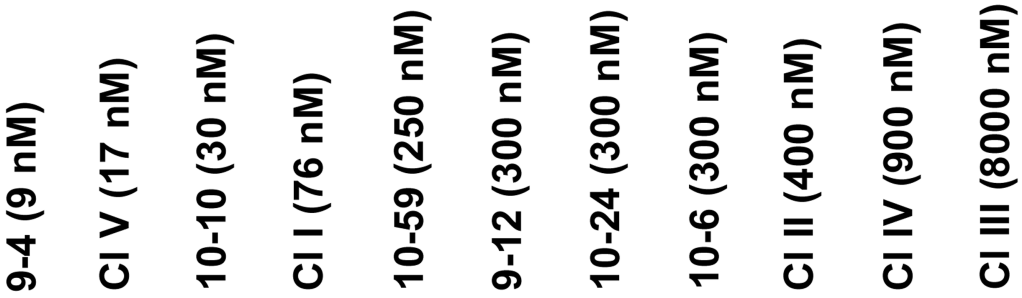

$$
\begin{aligned}
& \text { Aptamer }\left(\mathrm{K}_{\mathrm{d}(\mathrm{GTP})}\right)
\end{aligned}
$$

Figure 3.

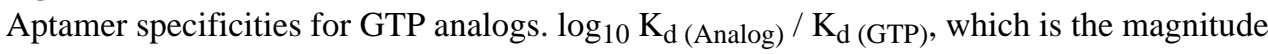
by which binding is specific for GTP compared to an analog, is plotted for each aptamer/ analog pair. The aptamers are arranged from left to right in order of affinity for GTP (shown in parentheses). For each aptamer, the number of analogs to which no binding was observed is represented in the bar graph at the top. 


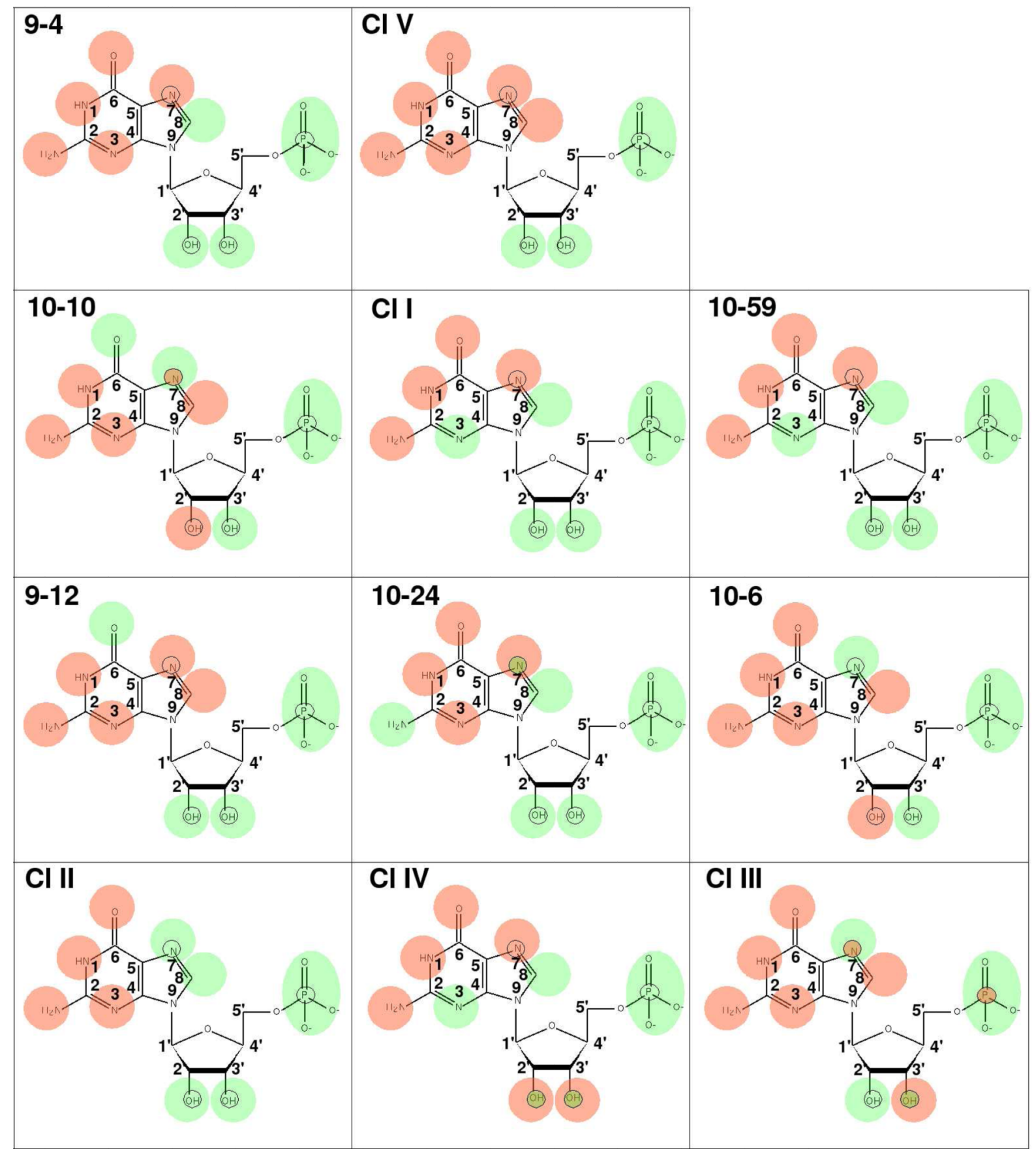

Figure 4.

Each aptamer has a unique pattern of binding specificity. For each aptamer, ligand positions where chemical modification resulted in large reductions in aptamer binding affinity relative to GTP (i.e. $\log _{10} \mathrm{~K}_{\mathrm{d} \text { (Analog) }} / \mathrm{K}_{\mathrm{d}(\mathrm{GTP})}>2$ ) are colored red. Ligand positions where chemical modifications did not reduce binding affinity more than 100 fold relative to GTP are colored green. The large shaded circles represent results for analogs with bulky modifications while the smaller inset circles represent results obtained with modified or deleted functional groups. Only the GMP moiety of the ligand is shown here because none 
of the modifications to the ligand $\beta$ or $\gamma$ phosphates that we tested resulted in substantiallyreduced binding affinity relative to GTP. Also see Supporting Figure 2. 


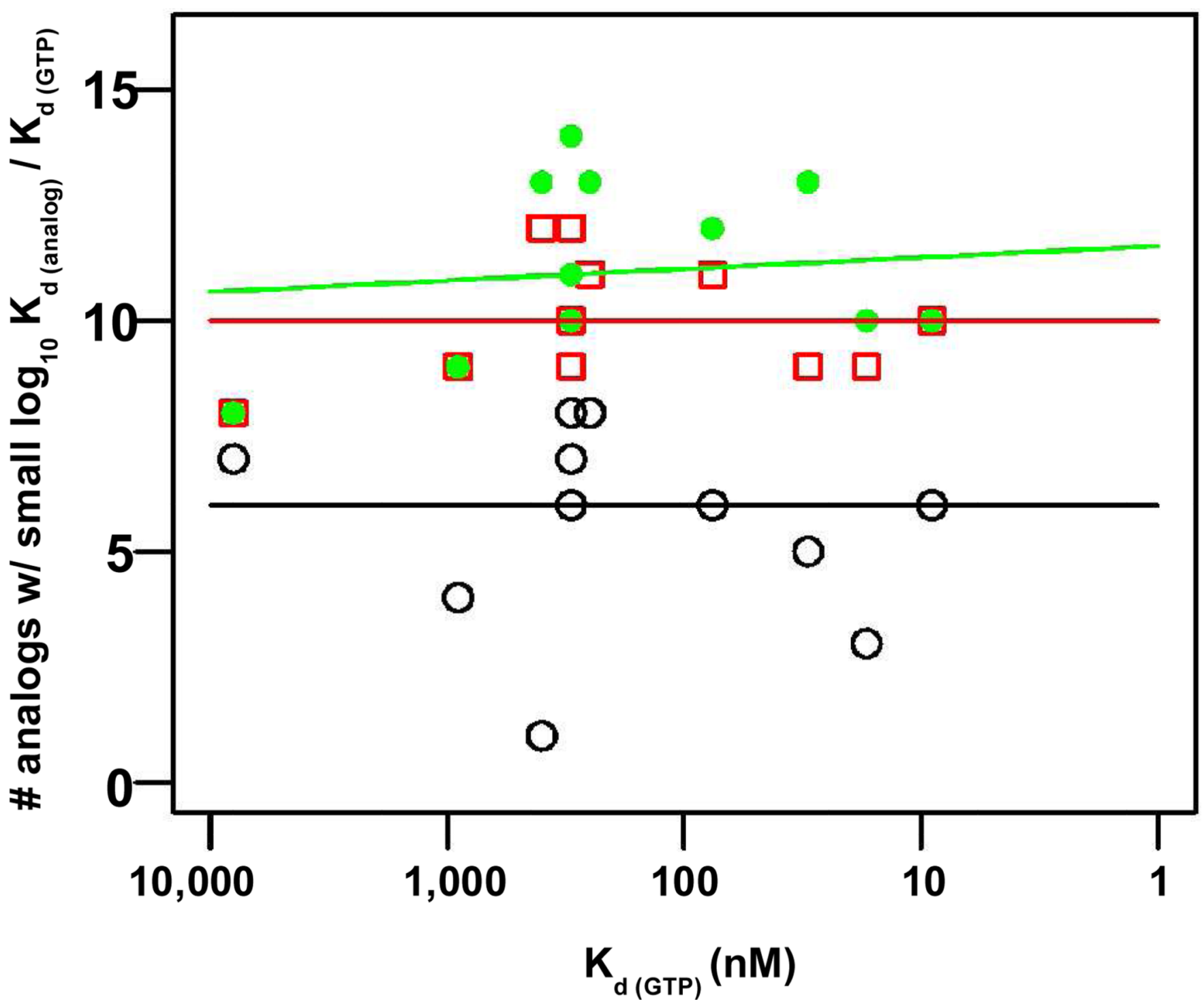

Figure 5.

Higher affinity aptamers are not more specific. For each aptamer, the number of analogs for

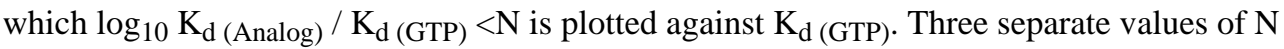
were employed to visualize different degrees of specificity: $\log _{10} \mathrm{~K}_{\mathrm{d} \text { (Analog) }} / \mathrm{K}_{\mathrm{d} \text { (GTP) }}<1$ (open black circles), <2 (open red squares), and $<3$ (solid green circles). The lines were generated by applying Kendall's robust line-fit method ${ }^{19}$ to the data indicated by the respective colors. Note that several of the plot points are the same for different values of $\mathrm{N}$, resulting in overlap between red squares and solid green circles. 
A.

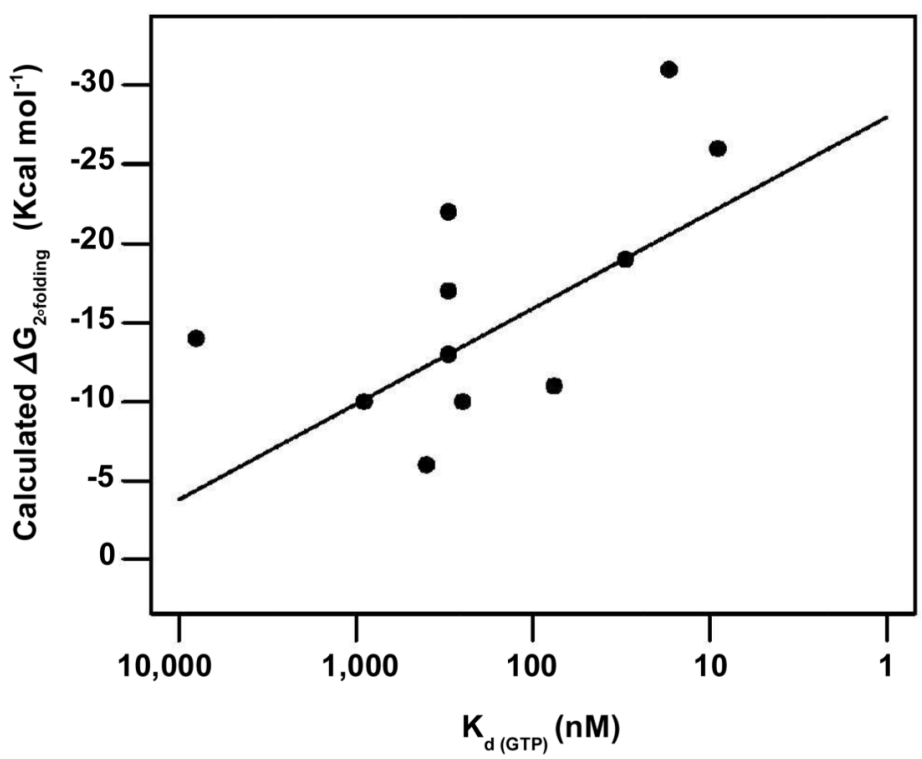

B.

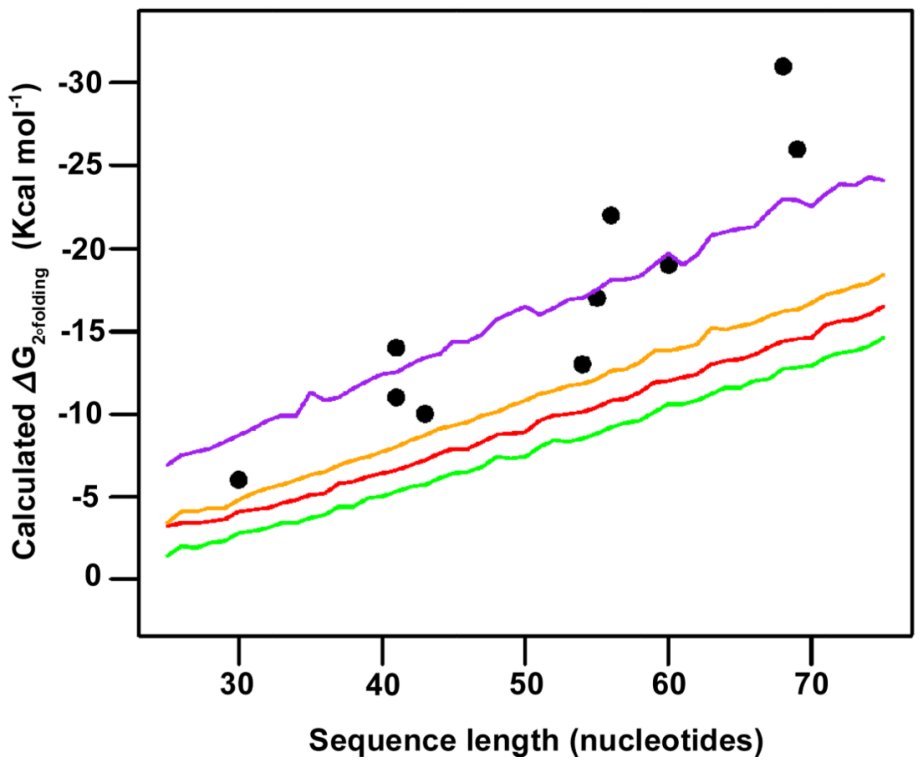

Figure 6.

Aptamer secondary structures are not unusually stable. (A) Free energy of secondary structure formation $\left(\Delta \mathrm{G}_{2}{ }^{\circ}\right.$ folding $)$ calculated for each aptamer using the program RNAeval

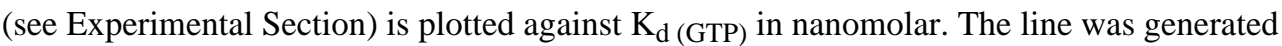
by applying Kendall's robust line-fit method. (B) Calculated $\Delta \mathrm{G}_{2}{ }^{\circ}$ folding for each aptamer is plotted against aptamer sequence length (solid black circles). The red line indicates the median $\Delta \mathrm{G}_{2}{ }^{\circ}$ folding values of 1000 random sequences plotted as a function of sequence 
length; the $33^{\text {rd }}, 67^{\text {th }}$, and $95^{\text {th }}$ percentiles are signified by the green, orange and purple lines, respectively. 


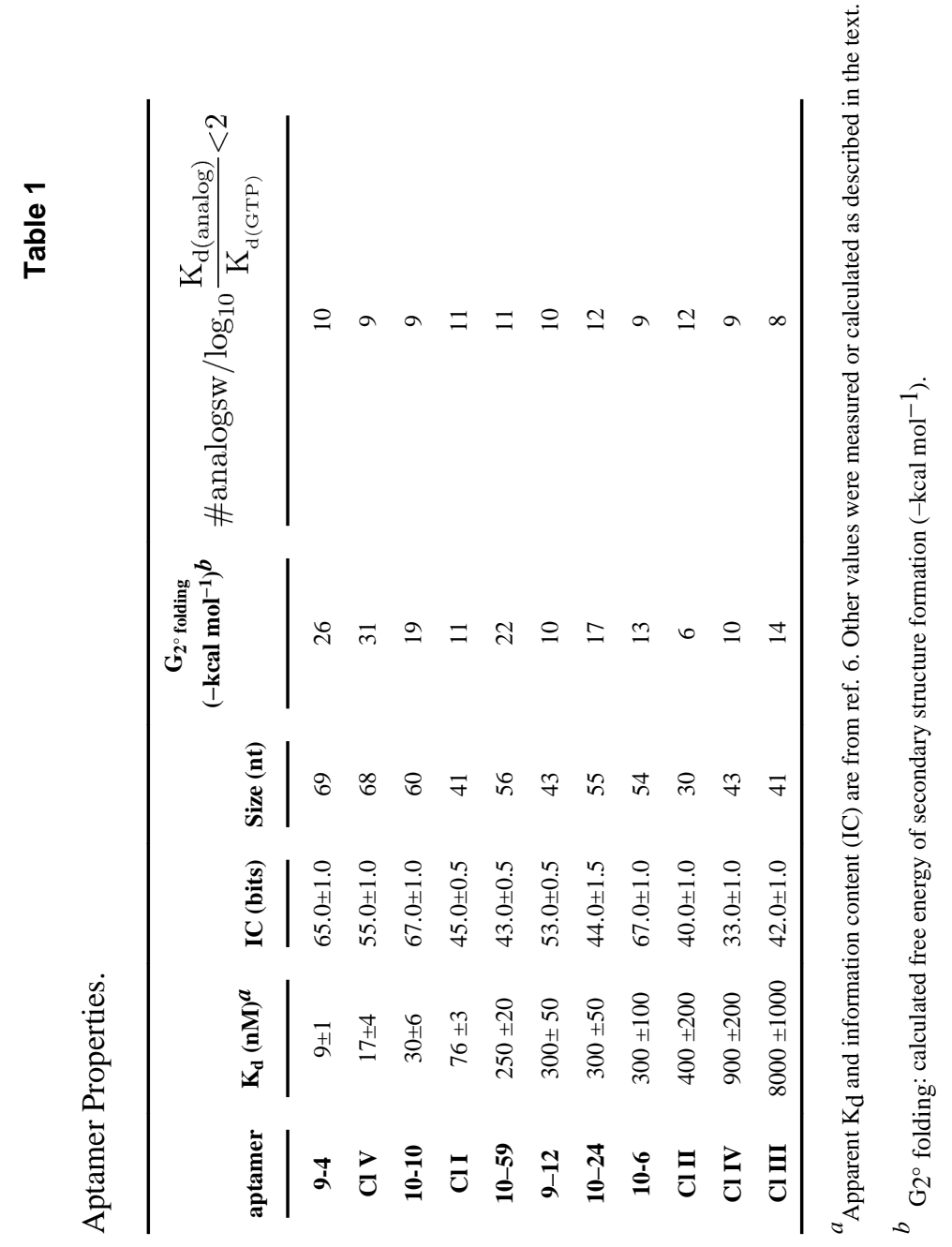

$J$ Am Chem Soc. Author manuscript; available in PMC 2015 January 09. 


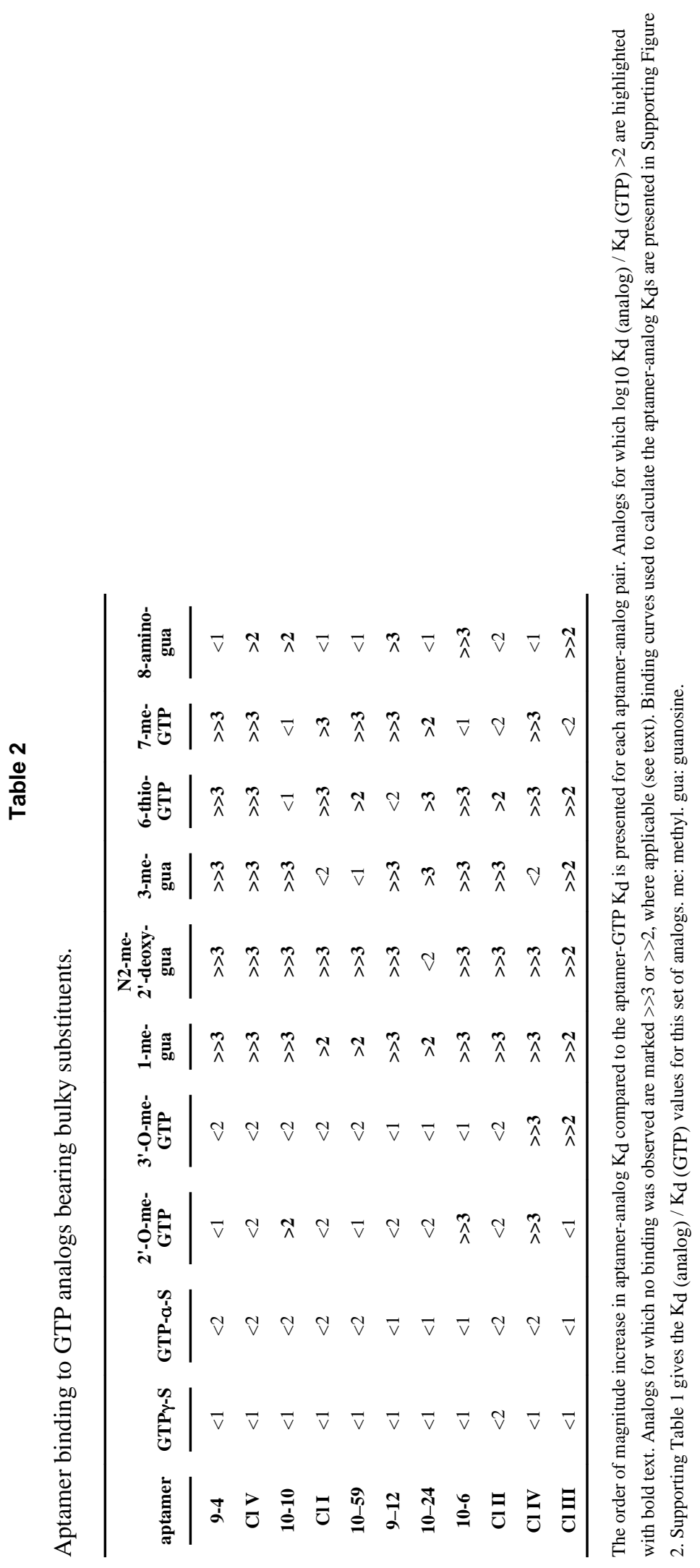




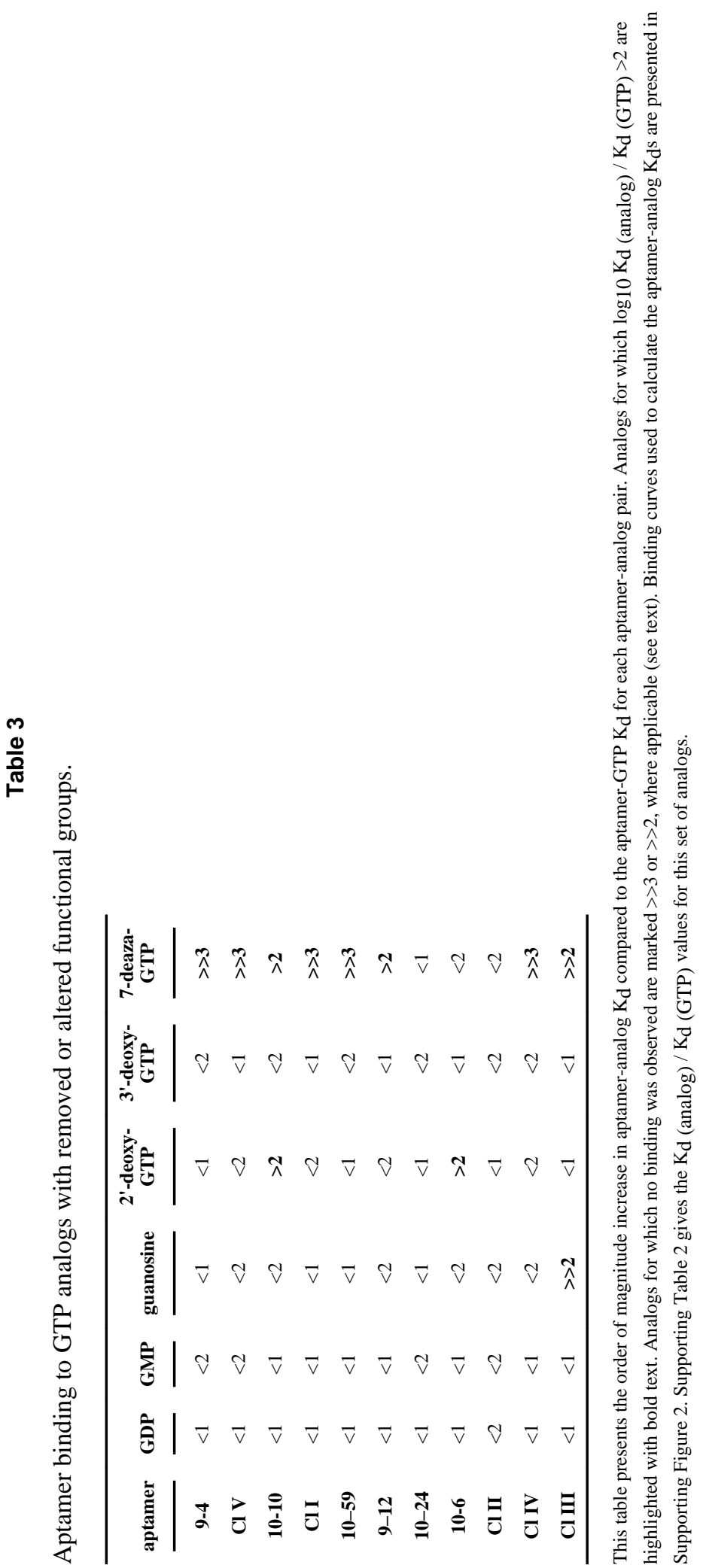

